\title{
$\mathrm{Wi}-\mathrm{Fi}$ 방송 서비스를 위한 방송 패킷 전송률에 따른 버스트 손실 특성 분석
}

김 세 미*, 김 동 현", 김 종 덕

\section{Analysis of Bursty Packet Loss Characteristic According to Transmission Rate for Wi-Fi Broadcast}

\author{
요 약
}

Se-mi Kim*, Dong-hyun Kim, Jong-deok Kim ${ }^{\circ}$

IEEE 802.11 무선 랜 기반 방송 서비스를 제공 할 때 한정적인 무선 자원을 이용하여 다수의 사용자들에게 원 활하게 서비스하기 위해 유니캐스트 패킷 대신 방송 패킷을 이용한다. 방송 패킷은 일정한 대역폭을 사용하여 다 수의 사용자들에게 동시에 패킷을 전송 할 수 있지만 손실 복원이 어려운 단점 있기 때문에 손실 특성 분석을 통 한 효율적인 패킷 복원 방안이 요구 된다. 손실의 특성 중에서 일정 구간에서 다수의 패킷이 연속적으로 손실 되 는 구간이 있는데 이를 버스트 손실 구간이라고 한다. 평균 패킷 손실율을 가지더라도 랜덤 손실과 버스트 손실의 특성에 따라 구간별 손실에 차이가 발생하기 때문에 같은 손실 복원 기법을 적용하더라도 복원율의 차이가 발생 한다. 따라서 손실의 본질을 분석 하고 이를 고려한 손실 복원 방안에 대한 연구가 필요하다. 본 논문에서는 전송 률에 따른 Wi-Fi 방송 실험을 통해 생성된 실제 손실 패턴을 바탕으로 4-상태 마코프 모델을 이용하여 버스트 손 실의 특성을 분석 하였다.

Key Words : WLAN, Broadcast, Bursty packet loss, Markov Model

\section{ABSTRACT}

When the IEEE 802.11 wireless LAN-based broadcasting services, we use broadcast packets to broadcast multimedia contents to a large number of users using limited wireless resources. However, broadcast transmission is difficult to recover the loss packets compared with unicast transmission. Therefore, analysis of packet loss characteristics is required to perform efficient packet recovery. The packet loss in wireless transmissions is often bursty with high loss data rate. Even if loss patterns have the same average packet loss, they are different in the recovery rate of random loss and burst loss depending on the nature. Therefore, the analysis and research of the nature of the loss are needed to recover loss packets considering bursty characteristics. In this paper, we experimented Wi-Fi broadcast transmission according to transmission rate and analyzed bursty characteristics of loss patterns using 4-state markov model.

\section{I. 서 론}

최근 스마트폰, 태블릿 $\mathrm{PC}$, 노트북과 같은 무선 네트워크를 사용하는 이동 단말의 보급이 증가하면

※ 이 논문은 부산대학교 자유 과제 학술연구비(2년)에 의하여 연구되었음

- 주저자: 부산대학교 컴퓨터공학과 이동통신 연구실, kimshem@mobile.re.kr, 준회원

교신저자 : 부산대학교 컴퓨터공학과 이동통신 연구실, kimjd@pusan.ac.kr, 종신회원

* 부산대학교 컴퓨터공학과 이동통신 연구실, dhkim1106@mobile.re,kr, 정회원

논문번호 : KICS2012-12-579, 접수일자 : 2012년 12월 31일, 최종논문접수일자 : 2013년 6월 27일 
서 무선 네트워크를 이용한 고용량 멀티미디어 스 트리밍 서비스에 대한 수요가 증가 하고 있다. 무선 네트워크에서 멀티미디어 스트리밍 서비스를 제공하 기 위한 무선통신기술로는 3G, LTE(Long Term Evolution), IEEE 802.11 기반 무선 랜 등이 있다. $3 \mathrm{G}, \mathrm{LTE}$ 기술은 하나의 기지국으로 넓은 서비스 반 경을 지원하지만 전송속도가 무선 랜에 비해 비교 적 낮고 데이터 사용량에 따라 높은 비용이 발생한 다. 무선 랜은 기술에 따라 수백Mbps까지 전송속도 를 지원하면서 낮은 비용으로 방송(Broadcast) 서비 스가 가능하기 때문에 개인 방송 서비스를 운용하 고자 할 때 다른 통신기술에 비해 유리하다.

무선 랜을 이용하여 멀티미디어 스트리밍 서비스 를 제공 하고자 할 때 다수의 사용자에게 한정된 무선 자원을 사용하여 안정적으로 서비스를 제공 하는 것이 필요하다. 방송 패킷을 사용하는 $\mathrm{Wi}-\mathrm{Fi}$ 방송 시스템은 하나의 방송 패킷을 이용하여 다수 의 사용자에게 동일한 콘텐츠를 제공 할 수가 있기 때문에 사용자 수가 크게 늘어도 무선 자원 부족 문제가 발생하지 않는 장점이 있다. 그러나 무선 랜 환경에서는 노드들이 미디엄 접근을 위해 서로 경 쟁하는 방식을 갖기 때문에 노드가 무선망에 많이 존재하거나 각 노드에서 발생하는 트래픽이 증가할 경우 미디엄 접근을 위한 경쟁으로 인해 높은 패킷 손실이 발생 한다. 방송 패킷은 유니캐스트 패킷과 달리 MAC(Medium Access Control) 계층 에서 손실 패킷을 재전송 하지 않기 때문에

FEC(Forward Error Correction)와 같이 응용 계층 에서 손실된 패킷을 복원 하는 기법을 적용 하는 것이 필요하다 ${ }^{[1,2]}$.

일반적으로 $\mathrm{FEC}$ 와 같은 손실 패킷 복원 기법을 적용 할 때 전체 손실율을 기반으로 여분 패킷의 비율을 결정한다. 하지만 실제 무선 랜 환경에서는 전송 중에 데이터가 갑자기 집중적으로 손실 되는 버스트(Burst) 손실이 발생 한다 ${ }^{[3,4]}$. 그림 1은 무선 랜 환경에서 발생 하는 버스트 패킷 손실과 랜덤 패킷 손실에 대한 개념을 비교 한 그림이다.

그림 1 에서 확인 할 수 있듯이 두 손실 데이터는 같은 수의 패킷이 손실 되었지만 손실이 발생하는 특성이 다르다. 버스트 손실이란 일반적으로 어떤 특정한 시간에 다수의 패킷이 손실 되어 높은 손실 율을 보이는 구간을 뜻한다. 즉, 다른 구간에 비해 서 패킷 손실율이 상대적으로 높은 구간을 버스트 손실 구간이라고 한다. 동일한 평균 패킷 손실율에

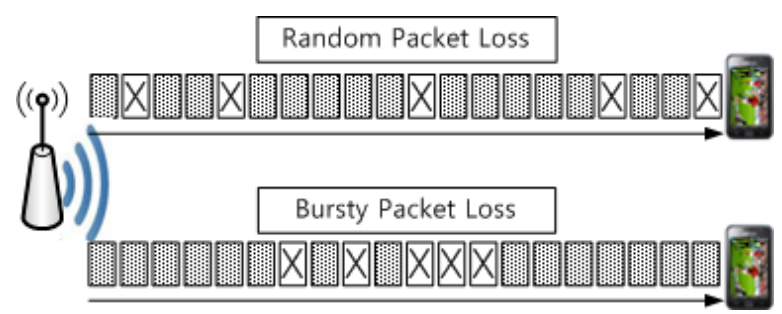

그림 1. 버스트 패킷 및 랜덤 패킷 손실 개념

Fig. 1. The concept of burst packet loss and random packet loss

서 같은 FEC 비율을 적용 할 때 버스트 손실과 랜 덤 손실의 특성에 따라 복원율의 차이가 발생 한다. 따라서 Wi-Fi 방송 시스템을 제공하기 위해 실제 실험을 통해 방송 패킷의 손실 특성을 분석 하고 그 특성에 따라 손실을 복원하는 방안에 대한 연구 가 필요하다.

본 논문에서는 IEEE 802.11 기반의 무선 랜 환 경의 방송 패킷 손실 특성을 확인하는 것을 목표로 한다. 전송률에 따라 감도의 차이가 있기 때문에 전 송률에 따라 무선 랜 손실 특성에 차이가 발생 할 것이라 예상 하고 전송률에 따른 방송 실험을 진행 하였다. 또한, 실험을 통해 발생한 실제 손실 데이 터를 기반으로 전송률에 따른 무선 랜 방송 손실 특성을 분석하였다. 손실을 분석하기 위해 4-상태 마코프 모델을 이용하여 버스트 특성 지표를 도출 하고 이를 기반으로 확률적인 접근을 통해 실제 방 송 패킷 손실 특성이 버스트 손실 특성을 가지는지 를 확인 하였다.

논문의 구성은 다음과 같다. 2장에서는 버스트 손실을 고려한 선행 연구와 버스트 특성을 분석하 기 위한 손실 분석 모델 및 특성 지표에 대해서 설 명한다. 3장에서는 랜덤 손실 패턴과 버스트 손실 패턴에 따른 복원율의 차이를 보이고 실제 방송 서 비스를 제공하기 위해서 방송 패킷 손실 특성 분석 및 이를 고려한 연구의 필요성에 대해서 이야기한 다. 4장에서는 전송률에 따른 IEEE 802.11 기반의 방송 손실 특성을 분석하기 위한 실험 환경 및 방 법을 설명하고 실험 결과를 분석한다. 또한 실제 방 송 패킷의 특성을 확률적으로 분석 하고 버스트 특 성을 고려한 $\mathrm{FEC}$ 와 평균 손실을 고려한 $\mathrm{FEC}$ 를 적 용 하여 성능을 평가한다. 마지막으로 5 장에서 본 연구의 결론을 맺고 향후 연구 방향을 제시한다.

\section{II. 관련 연구}

\section{1. 버스트 손실 분석 모델}


버스트 손실의 특성을 분석하기 위해서는 버스트 손실에 대한 정의 및 분석 방안이 필요하다. 본 논 문에서는 IETF(Internet Engineering Task Force) 의 RFC(Request for Comments) 3611 - RTCP-XR (RTP Control Protocol Extended Reports)에서 VoIP(Voice over IP)의 음성 트래픽을 모니터링 하 기 위해 정의한 버스트와 공백(Gap)의 개념을 이용 한다 ${ }^{[3,4]}$.

RTCP-XR 에서는 버스트 구간을 결정하는 최소 값인 $\mathrm{Gmin}$ 을 이용하여 버스트 구간을 정의한다. $\mathrm{Gmin}$ 이란 손실 패킷과 손실 패킷 사이에서 연속 적으로 정상 수신 될 수 있는 최대한의 패킷 수이 다. 즉, 손실 패킷과 손실 패킷 사이에 연속적으로 정상 수신 된 패킷이 $\mathrm{Gmin}$ 값 보다 작을 때 그 구 간을 버스트 구간이라고 하고 버스트 구간 이외의 나머지 구간은 공백 구간이 된다.

실제 손실 데이터를 바탕으로 손실을 분석하기 위해 분석 모델이 필요하다. 일반적으로 손실 분석 을 하기 위해서 평균 손실율과 길버트(Gilbert)모델 을 이용한다. 평균 손실율은 전체 패킷 중 손실 된 패킷의 비율을 나타낸 것으로 가장 많이 쓰이는 손 실 분석 지표이다. 길버트 모델은 2-상태 마코프 모 델과 같은 개념으로서 두 가지 상태를 가진다. 상태 0 은 정상적으로 수신 된 상태, 상태 1 은 패킷이 손 실 된 상태이며 각 상태에서 다른 상태로의 전이 확률을 통해 손실 데이터를 생성 할 수 도 있고 손 실 데이터를 바탕으로 각각의 패킷에 상태를 할당 하여 손실을 분석 할 수 있다. 하지만 두 가지 상 태를 가질 때는 연속적으로 손실이 일어나는 경우 만 버스트 구간이 되기 때문에 실제 버스트 손실 구간을 명확하게 구분하기 어렵다.

버스트 손실 구간과 공백 구간을 구분하여 손실 을 분석하기 위해서 우리는 다음 그림 2와 같은 4상태 마코프 모델[]을 이용한다.

4-상태 마코프 모델은 2-상태 마코프 모델과 유 사하지만 손실 구간 내에서도 $\mathrm{Gmin}$ 값 보다 적은 연속적인 정상 수신 상태가 존재하고 공백 구간에 서도 상태 4와 같이 고립된 손실이 발생할 수 있다. 즉, 버스트 구간과 공백 구간으로 나뉘며 각각의 구 간 내에서 손실과 수신 상태를 가진다. 각 상태에 대한 정의는 다음과 같다 ${ }^{[8]}$.

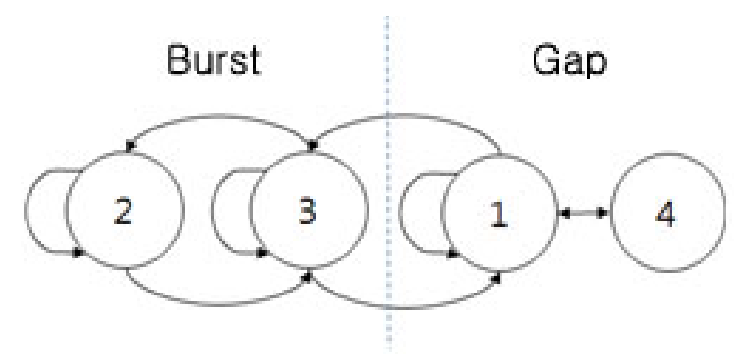

그림 2. 4-상태 마코프 모델

Fig. 2. 4-state Markov model

- 상태 1 - 공백 구간 내 패킷 수신

- 상태 2 - 버스트 구간 내 패킷 수신

- 상태 3 - 버스트 구간 내 패킷 손실

- 상태 4 - 공백 구간 내 하나의 손실 발생

본 모델을 이용하여 손실 데이터를 바탕으로 그 림 3 과 같이 각각의 패킷의 상태를 정의 할 수 있 으며 $\mathrm{Gmin}$ 값에 따라 버스트 구간과 공백구간을 나 눌 수 있다.

\begin{tabular}{|c|}
$\mid$ |--o000X000000X0000000X0XX000X0X0X0000000X0000000X00 \\
\hline 11111411111141111111323322232323111111141111111411 \\
\hline
\end{tabular}

그림 3. 4-상태 마코프 모델을 이용한 버스트 분석의 예 Fig. 3. The example of analysis of bursty packet loss using 4-state Markov model

그림 3은 $\mathrm{Gmin}$ 값이 5일 때 4-상태 마코프 모델 을 이용하여 손실 패턴을 분석 한 예이다. 첫 번째 손실 패킷과 두 번째 손실 패킷은 양 옆에 $\mathrm{Gmin}$ 값 이상의 연속적인 수신 패킷이 존재 하므로 첫 번째, 두 번째 손실 패킷은 공백 구간 내 고립 손 실인 상태 4 가 되고 나머지 정상 수신 상태는 모두 1 로 표시된다. 세 번째 패킷이 손실 되고나서 네 번째 패킷이 손실 될 때 세 번째 손실이 버스트 손 실에 속하는 상태 3 임을 알게 되고 그 사이에 정상 수신 된 패킷은 상태 2 가 된다.

본 손실 모델을 통해 분석한 내용을 바탕으로 IETF RFC 3611에서 정의한 버스트와 공백 구간의 길이, 밀도, 비율 등을 고려하여 버스트 손실을 분 석 할 수 있다. 각각의 분석 지표는 다음과 같다.

- $\mathrm{BL}(\mathrm{Burst}$ Length) : 버스트 구간 평균 길이

- $\mathrm{BD}$ (Burst Density) : 버스트 구간 내 평균 손 실 패킷 밀도 
- $\mathrm{BR}($ Burst Rate) : 전체 손실 패킷 중 버스트 손실 패킷 비율

- $\mathrm{GL}(\mathrm{Gap}$ Length) : 공백 구간 평균 길이

- $\mathrm{GD}(\mathrm{Gap}$ Density) : 공백 구간 내 손실 패킷 밀도

그림 3에 대한 지표 값은 표 1과 같이 나타낼 수 있다. 본 논문에서는 4-상태 손실 모델과 다섯 가지 지표를 이용하여 손실 특징을 분석 하고 실제 $\mathrm{Wi}-\mathrm{Fi}$ 방송 패킷 전송 중 버스트 패킷 손실의 발생 여부 확인한다.

표 1. 버스트 손실 지표를 이용한 분석의 예 Table 1. The example of analysis of bursty characteristics using burst loss value

\begin{tabular}{|c|c|c|c|c|c|}
\hline & $\begin{array}{c}\text { Burst } \\
\text { Length } \\
\text { (pkts) }\end{array}$ & $\begin{array}{c}\text { Burst } \\
\text { Density } \\
(\%)\end{array}$ & $\begin{array}{c}\text { Burst } \\
\text { Rate } \\
(\%)\end{array}$ & $\begin{array}{c}\text { Gap } \\
\text { Length } \\
\text { (패킷 } \\
\text { 수) }\end{array}$ & $\begin{array}{c}\text { Gap } \\
\text { Density } \\
(\%)\end{array}$ \\
\hline $\begin{array}{c}\text { 2-state } \\
\text { Markov } \\
\text { Model }\end{array}$ & 1.11 & 100 & 100 & - & - \\
\hline $\begin{array}{c}\text { 4-state } \\
\text { Markov } \\
\text { Model }\end{array}$ & 12 & 50 & 60 & 19 & 10.53 \\
\hline
\end{tabular}

\section{2. 기존 연구 분석}

무선 랜 환경에서 발생하는 버스트 손실과 관련 된 다양한 분야의 선행 연구들이 진행 되고 있다. 영상, $\operatorname{VoIP}(V o i c e$ over IP), 방송과 같은 다양한 멀 티미디어 서비스를 제공 할 때 버스트 패킷 손실이 발생 하는 것에 대해 주목 하고 이러한 버스트 패 킷 손실이 품질에 미치는 영향에 대한 관심이 높다. 관련 연구 ${ }^{[7,8]}$ 에서는 무선 랜 환경에서 멀티미디 어 데이터 전송을 할 때 발생하는 패킷 손실이 영 상의 품질에 미치는 영향에 대한 연구이다. 이 때 랜덤 패킷 손실과 버스트 패킷 손실로 인한 영상 품질 저하의 차이가 발생 하는 것을 알 수 있다. 또한, 관련 연구 ${ }^{[9,10]}$ 에서는 영상에서 발생하는 패킷 손실을 복원하기 위해 지연 시간이나 $\mathrm{FEC}$ 기법을 적용하여 패킷 손실을 복원 하는 방안을 제안한다. 하지만 관련 연구들은 시뮬레이션을 통한 분석이므 로 실제 손실 특성을 고려한 분석 결과가 아니며 손실 복원 기법 또한 버스트 손실 특성을 고려한 복원 기법이 아니다.

본 논문은 IEEE 802.11 무선 랜 환경에서 비디
오를 전송 할 때 발생하는 버스트 손실을 고려한 적응적 $\mathrm{FEC}$ 기법을 제안한 관련연구 ${ }^{11,12]}$ 를 기반으 로 연구를 진행 하였다. 본 논문에서는 무선 랜 환 경에서 발생하는 버스트 손실에 대해 버스트 손실 특성 지표를 바탕으로 버스트 손실을 분석 하고 특 성 지표를 이용하여 손실 복원 기법을 제안하였다. 본 논문에서는 손실 패킷을 효율 적으로 복원하기 위해 FEC 기법 중 Reed-Solomon Erasure Coding 을 사용 한다. 그리고 $\mathrm{FEC}$ 인자 $\mathrm{FEC}(\mathrm{n}, \mathrm{k})$ 의 원본 패킷과 여분 패킷의 비율을 결정 할 때 버스트 손 실 지표인 버스트 구간 평균 길이와 버스트 밀도 등을 활용하여 FEC 인자를 결정 한다. 본 관련연구 에서 제안하는 $\operatorname{FEC}(\mathrm{n}, \mathrm{k})$ 의 $\mathrm{FEC}$ 블록 크기 $\mathrm{n}$ 과 여 분 패킷 $\mathrm{n}-\mathrm{k}$ 는 다음 수식 (1)과 같다.

$$
\begin{gathered}
\text { XR: } \mathrm{n}=\text { BurstLenth }+ \text { Gmin } \\
\mathrm{n}-\mathrm{k}=\text { BurstLength * BurstDensity }
\end{gathered}
$$

$\mathrm{FEC}$ 블록 크기 $\mathrm{n}$ 은 평균 버스트 손실 길이에 $\mathrm{Gmin}$ 을 더한 값을 제안하는데 그 이유는 버스트 손실상태 일 때 버스트 손실 구간에서 벗어나기 위 해서는 최소 $\mathrm{Gmin}$ 값 이상의 패킷이 정상 수신 되 어야하기 때문에 평균 버스트 손실길이에 $\mathrm{Gmin}$ 값 을 더한 값을 $\mathrm{FEC}$ 블록 크기 $\mathrm{n}$ 으로 결정한다. 또 한 버스트 구간 내에 발생 하는 손실을 복원하기 위함 이므로 여분 패킷의 비율은 버스트 손실 구간 길이에 버스트 구간 내에 발생하는 손실 밀도를 곱 한 값을 여분패킷으로 제안하였다.

그러나 관련 연구에서는 $\mathrm{Gmin}$ 값을 $\mathrm{RFC} 3611$ 문서에서 권장하는 16 으로 고정 하여 버스트 손실 구간 길이와 버스트 구간 내 손실 밀도를 통해서 인자를 결정한다. 실제 방송 패킷 전송 과정에서 $15 \%$ 정도의 손실율을 가지는 패턴에서 $\mathrm{Gmin}$ 값이 10 이상이 되면 전체 손실 패킷 중 약 $90 \%$ 의 손실 패킷이 버스트 손실로 간주 되게 된다.

즉, $\mathrm{Gmin}$ 값이 16 이상 일 땐 손실 패킷 중 약 99\%의 손실 패킷이 버스트 손실로 간주되고 버스 트 길이 값이 증가 하여 거의 모든 패킷이 버스트 구간으로 판단되기 때문에 $5 \%$ 20\% 이내의 손실율 을 가지는 패턴에서 다양한 $\mathrm{Gmin}$ 값에 대해 버스트 특성을 분석하는 것이 필요하다. 또한 관련 논문은 $\mathrm{ns}-2$ 시뮬레이션을 통해 생성한 데이터를 기반으로 분석한 결과이기 때문에 실제 무선 랜 환경의 방송 손실 특성을 분석 하여 적용한 결과라고 보기 어렵 다. 
따라서 본 논문에서는 실제 무선 랜 방송 실험을 통해 무선 랜 환경에서 발생하는 전송 손실 특성을 $\mathrm{Gmin}$ 값에 따라 분석 하고 버스트 손실 지표를 활 용하여 버스트 손실 특성 발생 여부를 확인하고 실 제 방송 패킷의 손실 특성에 따라 복원 기법을 적 용 하는 것을 목표로 한다.

\section{III. 손실 특성 분석의 필요성}

일반적으로 $\mathrm{FEC}$ 를 적용 할 때 평균 손실율에 따 라 여분 패킷의 비율을 결정한다. 하지만 동일한 평 균 손실율을 가지는 패턴에 같은 FEC 비율을 적용 하더라도 손실이 발생하는 특성에 따라 복원율이 달라진다.

다음의 표 2는 동일한 손실율을 가지는 두 패턴 에 동일한 $\mathrm{FEC}$ 를 적용 했을 때 손실 패턴에 따른 복원 후 손실율을 비교 한 표이다. 실제 랜덤 손실 패턴과 버스트 손실 패턴에 따라 복원율에 차이가 발생 하는 것을 확인하기 위해 앞서 설명한 손실 모델을 이용하여 손실 패턴을 생성하였다. 전체 20000 개의 패킷 중 $5,10,15 \%$ 의 손실율을 가지는 랜덤 손실 패턴과 버스트 손실 패턴을 각각 생성 하여 동일한 FEC 비율을 적용 한 후 복원 후 손실 율을 구하였다. 본 표 2에서 PLR(Packet Loss Rate)은 평균 손실율, RPLR(Recovery Packet Loss Rate)은 복원 후 평균 손실율을 뜻하고 $\mathrm{R} / \mathrm{B}$ 의 $\mathrm{R}$ 은 랜덤 손실 패턴, $\mathrm{B}$ 는 버스트 손실 패턴을 뜻한다.

표 2. 손실 패턴에 따른 FEC 복원율 비교

Table 2. The comparison of FEC recovery rate according to packet loss pattern

\begin{tabular}{|c|c|c|c|c|c|c|}
\hline \multirow{2}{*}{ R/B } & \multirow{2}{*}{$\begin{array}{c}\text { PLR } \\
(\%)\end{array}$} & \multicolumn{5}{|c|}{ FEC } \\
\cline { 3 - 7 } & & FEC & FEC & FEC & FEC \\
& $(14,10)$ & $(13,10)$ & $(12,10)$ & $(11,10)$ \\
\hline R1 & 5 & 0 & 0 & 0 & 0 & 0.49 \\
\hline R2 & 10 & 0 & 0 & 0 & 0.06 & 2.32 \\
\hline R3 & 15 & 0 & 0 & 0.39 & 1.50 & 6.68 \\
\hline B1 & 5 & 0.37 & 1.26 & 1.76 & 2.59 & 3.48 \\
\hline B2 & 10 & 2.18 & 3.52 & 4.50 & 5.84 & 7.45 \\
\hline B3 & 15 & 4.21 & 6.05 & 7.38 & 8.91 & 11.16 \\
\hline
\end{tabular}

표 2에서 확인 할 수 있듯이 동일한 손실율을 가 지더라도 랜덤 손실에 비해 버스트 손실 발생 할 경우 같은 비율의 $\mathrm{FEC}$ 를 적용 했음에도 불구하고 손실 패킷 복원율에 차이가 발생하는 것을 확인 할
수 있다. 일반적으로 손실된 패킷을 복원하기 위해 $\mathrm{FEC}$ 의 비율을 정할 때 평균 패킷 손실율을 고려하 여 여분 패킷의 비율을 정하는 방법을 사용한다. 원 본 패킷의 비율을 10 으로 정하고 여분 패킷의 비율 을 1 에서 5 로 증가 시켰을 때 랜덤 손실에 비해 버 스트 손실의 복원이 제대로 이루어지지 않는 것을 볼 수 있다.

표 2의 $10 \%$ 의 손실을 가지는 $\mathrm{R} 2, \mathrm{~B} 2$ 패턴을 비 교 해 볼 때 전체 10 개의 패킷 중 $10 \%$ 의 패킷 손 실이 발생 될 것을 고려하여 $\operatorname{FEC}(12,10)$ 을 적용하 여 2 개의 여분 패킷을 덧 붙여 전송 하였을 때 랜 덤 손실 패턴의 경우 복원 후 손실 율이 $0.06 \%$ 로 복원율이 높지만 버스트 손실 패턴의 경우에는 $5.84 \%$ 로 절반도 채 복원 되지 못한 것을 알 수 있 다. 즉, 실제 무선 랜을 통해 방송을 제공 하고자 할 때 버스트 패킷 손실이 발생 할 경우 손실 특성 을 고려하지 않은 평균 패킷 손실율에 따른 복원 방법은 비효율적임을 확인 할 수 있다.

실제로 2장에서 설명했던 수식 (1)을 이용하여 버스트 손실 특성을 고려한 $\mathrm{FEC}$ 를 적용 하는 방법 이 효율 적인지 확인하기 위하여 표 2의 R2, B2 패턴에 수식 (1)을 적용 하여 FEC 비율을 결정 하 면 다음 표 3 과 같다.

표 3. 버스트 특성을 고려한 FEC 복원율 비교 Table 3. The comparison of FEC recovery rate according to burst loss characteristics

\begin{tabular}{|c|c|c|c|c|c|c|}
\hline $\mathrm{R} / \mathrm{B}$ & Gmin & $\mathrm{BL}$ & $\mathrm{BD}$ & $\mathrm{n}$ & $\mathrm{n}-\mathrm{k}$ & $\begin{array}{c}\text { RPLR } \\
(\%)\end{array}$ \\
\hline \multirow{3}{*}{$\mathrm{R} 2$} & 3 & 3.15 & 63.41 & 6 & 2 & 0 \\
\cline { 2 - 7 } & 9 & 13.43 & 22.16 & 22 & 3 & 0.12 \\
\cline { 2 - 7 } & 15 & 130.84 & 11.52 & 146 & 15 & 0.23 \\
\hline \multirow{3}{*}{$\mathrm{B} 2$} & 3 & 8.00 & 68.55 & 11 & 5 & 1.65 \\
\cline { 2 - 7 } & 9 & 35.79 & 42.83 & 57 & 17 & 0.57 \\
\cline { 2 - 7 } & 15 & 42.76 & 40.54 & 56 & 17 & 1.53 \\
\hline
\end{tabular}

표 3은 R2, B2 패턴의 버스트 손실 특성 지표를 활용하여 $\mathrm{FEC}$ 블록 크기와 여분 패킷의 비율을 결 정 하고 이러한 비율을 적용 하였을 때 복원 후 손 실율을 나타낸 것이다. $\mathrm{Gmin}$ 값에 따라 버스트 손 실 특성 지표의 크기가 달라지기 때문에 FEC 비율 도 달라지는 것을 확인 할 수 있다. 랜덤 손실 패 턴의 경우 $\mathrm{Gmin}$ 값이 9에서 15로 증가함에 따라 버 스트 길이가 증가하면서 $\mathrm{FEC}$ 비율이 급격 하게 변 하는 것을 볼 수 있다. 하지만 버스트 손실의 경우 
실제 일정한 구간에 다수의 손실이 일어나는 특성 이 있기 때문에 일정 $\mathrm{Gmin}$ 값 내에서는 따라 버스 트 길이와 $\mathrm{FEC}$ 비율이 급격 하게 변하지 않는 것 을 확인 할 수 있다.

두 패턴 모두 수식(1)을 적용하여 $\mathrm{FEC}$ 를 적용 하였을 때 복원 후 손실율은 $2 \%$ 미만으로 $80 \%$ 정 도의 손실을 복원 한 것을 확인 할 수 있지만 FEC 블록의 크기와 여분 패킷의 비율이 달라지기 때문 에 본 기법을 사용 할 경우 방송 환경에 맞는 적절 한 FEC 비율을 결정해야 한다. 안정적인 방송을 제 공하기 위해서는 실제 방송 전송 과정에서 발생하 는 손실 특성을 분석 하고 랜덤 손실 특성을 보이 는 경우와 버스트 손실 특성을 구분 하여 각 특성 을 고려한 효율적인 손실 복원 방안에 대한 연구가 필요 하다.

본 논문에서는 실제 $\mathrm{Wi}-\mathrm{Fi}$ 방송 패킷 전송 과정 에서 발생 하는 손실 특성이 버스트 손실 특성인지 를 확인하기 위해 각각의 버스트 특성 지표를 구하 고 확률적인 접근을 통해 손실 특성을 분석하는 것 을 목표로 한다. 또한 실제 방송 패킷의 손실 특성 에 따라 FEC 기법을 적용 하고 효율을 알아본다.

\section{IV. 실험 및 결과 분석}

\section{1. 실험 환경}

본 논문에서는 Wi-Fi 방송 시스템의 전송률에 따 른 버스트 특성을 알아보기 위해 테스트 베드를 구 성 하였다. 테스트 베드는 방송 서버, 방송 $\mathrm{AP}$, 그 리고 방송 수신을 위한 수신 단말기로 구성 된다. 서버와 $\mathrm{AP}$ 는 유선으로 연결하고 단말기는 무선구간 을 통해 데이터를 전송 받는다.

하드웨어는 Alix(AMD Ceode LX based system boards) 임베디드 보드를 사용 하였고 테스트베드 구성 환경에 따른 불안정한 링크의 품질을 높이기 위해 Omni 안테나를 사용하였다. IEEE $802.11 \mathrm{~g}$ 무 선 랜 기술을 지원하는 Atheros 칩셋 무선 랜 카드 를 사용 하였다. 단말기의 랜 카드는 노트북 내장 칩셋인 Intel(R) PRO/Wireless 3945ABG Network Connection을 이용하였다.

소프트웨어는 Pyramid Linux kernel 2.6.19.2을 이용하였고 $\mathrm{MadWiFi}$ 0.9.4를 수정하여 전송률을 $1-54 \mathrm{Mbps}$ 로 변경 하여 전송 할 수 있도록 하였다. 또한 실제 실험을 통해 발생한 손실 패턴을 이용하 여 버스트 손실에 대해 분석하기 위해 프로그램을 개발하였다. 프로그램은 C언어 기반으로 일반 손실,
길버트 모델, 4-상태 마코프 모델을 이용하여 실제 데이터를 분석 할 수 있고 각각의 모델과 확률을 통해 손실 패턴을 생성 할 수 있도록 구현 하였다. 본 프로그램은 2장에서 설명했던 것처럼 각 패킷의 상태를 할당하여 버스트 구간과 공백 구간을 결정 하고 각각의 구간에 대한 손실 특성 지표를 도출 할 수 있다.

\section{2. 실험 내용}

IEEE $802.11 \mathrm{~g}$ 에서는 $1-54 \mathrm{Mbps}$ 무선 랜 전송률 을 이용할 수 있다. 하지만 현재 상용 $\mathrm{AP}$ 의 경우 방송 패킷의 전송 속도가 $1 \mathrm{Mbps}$ 로 고정 되어있다. 전송률에 따른 감도의 차이가 발생하기 때문에 전 송률에 따른 손실 특성에 차이가 발생 할 것을 기 대하고 전송률을 다양하게 변경 할 수 있도록 테스 트베드를 구현 하였다.

본 논문에서는 방송 패킷의 무선 랜의 물리적 전 송률에 따른 버스트 손실 특성을 분석하기 위해 $1 \mathrm{Mbps}$ 부터 $54 \mathrm{Mbps}$ 까지 전송률에 따른 패킷 전송 실험을 하였다. 실험결과 전송률이 높아질수록 $20 \%$ 이상의 손실율을 보였으며 이 때 $\mathrm{Gmin}$ 값이 높아지 면 $90 \%$ 이상의 패킷이 버스트 구간으로 간주되는 것을 확인하였다. 따라서 약 5\% 20\% 사이의 손실 율을 가지는 $1-18 \mathrm{Mbps}$ 사이의 결과 값에 대해서만 버스트 특성을 분석하였다.

본 실험은 전송률에 따른 버스트 손실 특성을 알 아보기 위한 실험이므로 다른 전송 인자들은 고정 값으로 실험을 진행하였다. 패킷의 Payload 크기는 1024byte로 고정 하고, 방송 트래픽의 평균 데이터 률은 $800 \mathrm{kbps}$ 를 기본으로 설정하였다. 실험은 다른 트래픽의 간섭을 피하기 위해 심야시간대의 실험실 내에서 진행되었으며 평균 기본 트래픽은 $100 \mathrm{kbps}$ 이하의 값을 보였다. $\mathrm{AP}$ 와 노트북의 거리는 직선거 리 50M이며 실험은 전송률에 따라 각각 30 분씩 10 회씩 실험 되었다. 각각의 값들은 10 회 실험의 평 균값을 나타낸 것이다.

\section{3. 실험 결과}

전송률에 따른 $\mathrm{Wi-Fi}$ 방송 실험 결과 각각의 전 송률에 대해 $1 \mathrm{Mbps}$ 에서는 8.65 로 약 $8.5 \%, 6,12$, $18 \mathrm{Mbps}$ 에서는 각각 $15.48 \%, 16.19 \%, 16.44 \%$ 로 약 $16 \%$ 의 평균 손실율을 보였으며 전송률이 높아질수 록 손실율이 높아지는 것을 확인 할 수 있었다.

각 실험을 통해 생성된 손실 패턴을 분석하기 위 해서 4-상태 마코프 모델을 이용하여 버스트 특성 
분석 지표를 도출 하였다. 버스트 특성 분석 지표 값은 $\mathrm{Gmin}$ 값에 따라 달라진다. 따라서 본 논문에서 는 다양한 분석을 하기 위해 Gmin값을 1 부터 16 까 지 증가 시키면서 버스트 특성을 분석 하였다.

\subsection{1. 버스트 지표}

버스트 특성을 분석하기 위해 실제 손실 데이터 를 이용하여 $\mathrm{Gmin}$ 값에 따른 버스트 길이와 버스트 밀도 지표를 계산하면 다음 표 4와 같다.

표 4. 전송률과 $\mathrm{Gmin}$ 값에 따른 버스트 특성 지표

Table 4. The burst characteristic according to transmission rate and $\mathrm{Gmin}$

\begin{tabular}{|c|c|c|c|c|c|c|}
\hline \multirow{2}{*}{$\begin{array}{c}\text { Trans- } \\
\text { mission } \\
\text { Rate } \\
\text { (Mbps) }\end{array}$} & \multicolumn{3}{|c|}{ Burst Length(pkts) } & \multicolumn{3}{c|}{ Burst Density(\%) } \\
\cline { 2 - 7 } & 5 & 10 & 15 & 5 & 10 & 15 \\
\hline 1 & 4.54 & 14.24 & 26.07 & 47.80 & 25.37 & 19.01 \\
\hline 6 & 8.11 & 28.39 & 84.00 & 43.12 & 25.16 & 19.92 \\
\hline 12 & 9.02 & 28.65 & 85.13 & 43.81 & 27.94 & 22.11 \\
\hline 18 & 10.16 & 28.86 & 86.52 & 46.08 & 28.44 & 23.83 \\
\hline
\end{tabular}

표 4는 전송률에 따른 손실 패턴이 $\mathrm{Gmin}$ 값이 5 , 10,15 일 때의 평균 버스트 구간의 길이와 버스트 구간내의 평균 손실 패킷 밀도를 나타낸 값이다. 표 4 에서 볼 수 있듯 전송률에 따라 손실율의 차이가 발생하기 때문에 전송률에 따른 특성보다는 손실율 에 따라서 각각의 지표의 차이가 발생하는 것을 볼 수 있다.

약 $8.5 \%$ 의 손실율을 가지는 $1 \mathrm{Mbps}$ 의 경우 다른 $15 \%$ 정도의 손실율을 보이는 전송률에 비해서 낮은 버스트 손실 길이를 가지고 비슷한 손실율을 가지 는 경우에는 거의 유사한 값을 가지는 것을 확인 할 수 있었다. 버스트 밀도의 경우에는 전송률과 손 실율의 차이에 비해서 큰 차이를 보이지 않고 거의 유사한 값을 가짐을 알 수 있다.

\subsection{2. 공백 지표}

표 5 는 전송률에 따른 손실 패턴이 $\mathrm{Gmin}$ 값이 5 , 10,15 일 때의 평균 공백 구간의 길이와 평균 공백 구간 내 평균 고립 손실의 밀도를 나타낸 값이다. 앞선 버스트지표와 마찬가지로 전송률에 따라 손실 율의 차이가 발생하기 때문에 전송률에 따른 특성 보다는 손실율에 따라서 각각의 지표가 차이가 발 생하는 것을 볼 수 있다.
표 5. 전송률과 $\mathrm{Gmin}$ 값에 따른 공백 지표

Table 5. The gap characteristic according to transmission rate and $\mathrm{Gmin}$

\begin{tabular}{|c|c|c|c|c|c|c|}
\hline \multirow{2}{*}{$\begin{array}{c}\text { Trans- } \\
\text { mission } \\
\text { Rate } \\
\text { (Mbps) }\end{array}$} & \multicolumn{3}{|c|}{ Gap Length(pkts) } & \multicolumn{3}{c|}{ Gap Density(\%) } \\
\cline { 2 - 7 } & 5 & 10 & 15 & 5 & 10 & 15 \\
\hline 1 & 45.93 & 34.88 & 35.11 & 4.08 & 1.80 & 0.96 \\
\hline 6 & 19.20 & 18.79 & 22.31 & 3.81 & 0.92 & 0.23 \\
\hline 12 & 18.79 & 18.45 & 21.88 & 3.79 & 0.91 & 0.27 \\
\hline 18 & 17.56 & 18.02 & 21.41 & 3.68 & 0.90 & 0.26 \\
\hline
\end{tabular}

평균 공백 구간 길이는 버스트 손실과 반대로 손 실율이 낮을수록 길어지며 비슷한 손실율을 가지는 경우에는 유사한 값을 가지는 것을 확인 할 수 있 었다. 공백 밀도의 경우에는 고립 손실만을 포함하 기 때문에 전송률과 손실율의 차이에 비해서 큰 차 이를 보이지 않고 $5 \%$ 이하의 낮은 값을 가짐을 알 수 있다. 또한 $\mathrm{Gmin}$ 값이 커질수록 공백 구간에 속 하는 손실 패킷보다 버스트 손실에 속하는 손실 패 킷이 증가 하는 것을 알 수 있다.

$\mathrm{Gmin}$ 값에 따른 버스트 특성 분석 지표들의 변화 를 확인하기 위해서 다음 그림 4 와 같이 $\mathrm{Gmin}$ 값을 1 에서 16 까지 증가시켰을 때 지표 값을 나타내었다.

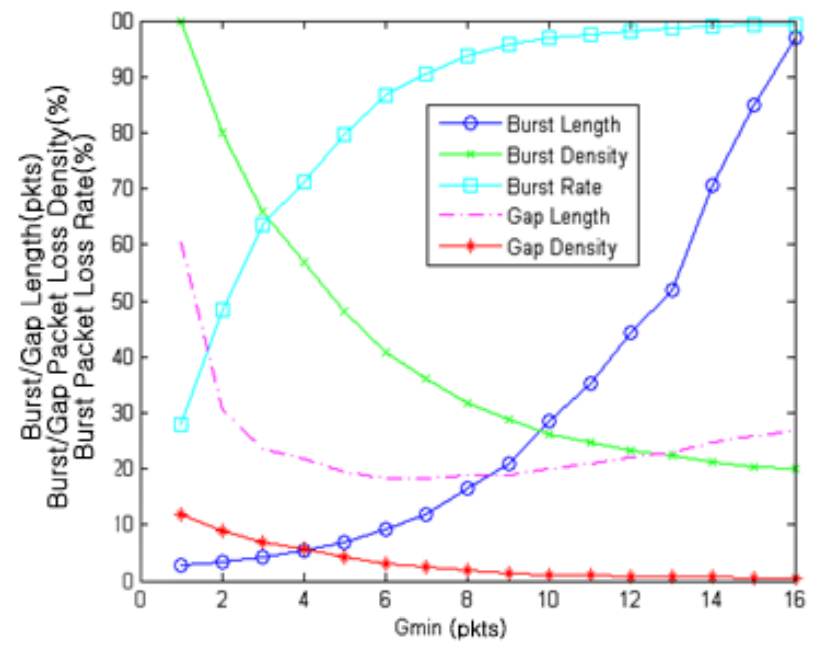

그림 4. $\mathrm{Gmin}$ 값에 따른 버스트 손실 특성 지표 변화(전송률=6Mbps, PLR $=15.48 \%$ )

Fig 4. The burst characteristic according to Gmin (Transmission Rate $=6 \mathrm{Mbps}, \mathrm{PLR}=15.48 \%$ )

그림 4에서 확인 할 수 있듯이 버스트 길이와 전 체 손실 패킷 중 버스트 손실 패킷의 비율은 $\mathrm{Gmin}$ 값이 커질수록 증가하고 버스트 구간 내 손실 밀도 와 공백 구간 내 고립 손실 밀도는 $\mathrm{Gmin}$ 값이 증가 
함에 따라 감소하는 것을 확인 할 수 있다. 또한 공백 구간 길이는 $\mathrm{Gmin}$ 값에 의해 차이 크게 발생 하지 않는 것을 확인 할 수 있다. 손실율에 따른 값 의 크기는 차이가 있었지만 전송률이 달라도 각 특 성의 증감 그래프의 형태는 유사함을 확인 하였다.

\section{4. 결과 분석}

실험 결과를 토대로 볼 때 전송률에 따른 특성 보다는 손실율 따라 버스트 지표 값이 달라지는 것 을 확인 하였다. 또한 $\mathrm{Gmin}$ 값에 의해 버스트 손실 지표가 결정 되는 것을 확인하였으나 실제 버스트 한 손실 특성을 보이는지에 대한 확인은 불가능하 다. 따라서 실제 무선 랜 환경에서 방송 패킷을 전 송하는 실험을 통해 생성 된 손실 패턴이 버스트한 특성을 가지는 지에 대한 여부를 분석하기 확률적 인 접근이 필요하다.

어떤 임의의 손실 패킷에 대하여 해당 패킷이 버 스트 손실로 평가될 확률을 토대로 실험결과가 버 스트 특성을 가지는지를 분석한다. 즉, 확률로 실제 Wi-Fi 방송 실험을 통해 나온 손실 결과가 버스트 특성을 보이는지 아니면 일반적인 랜덤 손실의 특 성을 보이는지에 대한 여부를 판단 할 수 있다.

어떤 임의의 손실 패킷이 버스트 손실로 평가되 기 위해서는 그 손실 패킷 앞으로 $\mathrm{Gmin}$ 값, 뒤로 $\mathrm{Gmin}$ 값 내에서 하나 이상의 손실이 발생 할 때 버스트 하다고 평가 된다. 따라서 앞, 뒤로 $\mathrm{Gmin}$ 값 이내에 손실이 존재 하지 않아서 버스트 손실로 평가 되지 않을 확률을 빼면 버스트 손실로 평가될 확률이라고 판단 할 수 있다. 즉, 임의의 손실 패킷 에 대하여 해당 패킷이 버스트 손실로 평가될 확률 인 $\mathrm{Pp}$ 를 수식으로 나타내면 다음 수식 (2)와 같다.

\section{$\mathrm{PB}=1-(1-\mathrm{P})(\mathrm{Gmin} * 2)$}

수식 (2)의 $\mathrm{P}$ 는 평균 손실율을 뜻한다. 즉, $1-\mathrm{P}$ 는 손실이 일어나지 않을 확률이므로 앞, 뒤로 $\mathrm{Gmin}$ 값 이내에 손실이 존재 하지 않아야 버스트로 판단되 기 때문에 $\mathrm{Gmin} * 2$ 만큼의 패킷이 손실이 일어나야 하지 않아야 한다. 수식 (2)을 이용하여 평균 손실 율에 따라 임의의 손실 패킷이 버스트 손실로 평가 될 확률을 계산하여 나타 낼 수 있다.

표 6은 평균 손실율에 따라 임의의 손실 패킷이 버스트 손실로 평가될 확률을 계산 하여 정리 한 것이며 이는 $\mathrm{Gmin}$ 따른 평균 버스트 비율과 같은 값을 가진다.
표 6. 임의의 손실 패킷이 버스트 손실로 평가 될 확률 Table 6. The probability of any loss packet is proved to bursty loss packet

\begin{tabular}{|c|c|c|c|}
\hline \multirow{2}{*}{$\begin{array}{c}\text { PLR } \\
(\%)\end{array}$} & \multicolumn{3}{|c|}{ PB (\%) } \\
\cline { 2 - 4 } & Gmin=5 & Gmin=10 & Gmin=15 \\
\hline 8 & 56.56 & 81.13 & 91.80 \\
\hline 9 & 61.06 & 84.84 & 94.09 \\
\hline 15 & 80.31 & 96.12 & 99.24 \\
\hline 16 & 82.51 & 96.94 & 99.46 \\
\hline
\end{tabular}

표 7. 실제 Wi-Fi 방송 손실 패턴의 $\mathrm{Gmin}$ 에 따른 평균 버 스트 비율

Table 7. The burst rate of Wi-Fi broadcast loaa pattern according to Gmin

\begin{tabular}{|c|c|c|c|c|}
\hline \multirow{2}{*}{$\begin{array}{c}\text { Trans- } \\
\text { mission } \\
\text { Rate } \\
\text { (Mbps) }\end{array}$} & PLR & \multicolumn{3}{|c|}{ Burst Loss Rate(\%) } \\
\cline { 3 - 5 } & $\%$ & Gmin=5 & Gmin=10 & Gmin=15 \\
\hline 1 & 8.65 & 56.94 & 81.71 & 93.52 \\
\hline 6 & 15.48 & 82.53 & 97.33 & 99.64 \\
\hline 12 & 16.19 & 84.16 & 97.55 & 99.68 \\
\hline 18 & 16.44 & 86.04 & 97.84 & 99.72 \\
\hline
\end{tabular}

표 7은 실제 Wi-Fi 방송 실험결과 값을 기반으 로 $\mathrm{Gmin}$ 값에 따라 평균 버스트 비율 값을 나타낸 표이다. 표 6의 값과 표 7의 값을 비교하였을 때 손실율에 따라 거의 유사한 값을 가지는 것을 확인 할 수 있다. 따라서 실제 무선 랜 환경의 W-Fi 방 송 패킷에서 발생하는 손실 패턴은 버스트 손실 특 성 보다는 랜덤 손실에 가까운 특성을 가지는 것을 확인 할 수 있다.

표 8. 실제 Wi-Fi 방송 손실 패턴의 버스트 특성을 고려한 FEC 복원율 비교

Table 3. The comparison of FEC recovery rate according to burst loss characteristics of Wi-Fi broadcast loss pattern

\begin{tabular}{|c|c|c|c|c|c|c|}
\hline $\begin{array}{c}\text { Trans- } \\
\text { mission } \\
\begin{array}{c}\text { Rate } \\
\text { (Mbps) }\end{array}\end{array}$ & Gmin & BL & BD & $\mathrm{n}$ & $\mathrm{n}-\mathrm{k}$ & $\begin{array}{c}\text { RPLR } \\
(\%)\end{array}$ \\
\hline \multirow{3}{*}{1} & 3 & 4.54 & 47.80 & 8 & 2 & 0.02 \\
\cline { 2 - 7 } & 9 & 14.24 & 25.37 & 23 & 4 & 0.24 \\
\cline { 2 - 7 } & 15 & 26.07 & 19.01 & 41 & 5 & 0.49 \\
\hline \multirow{3}{*}{12} & 3 & 9.02 & 43.81 & 12 & 4 & 0.68 \\
\cline { 2 - 7 } & 9 & 28.65 & 27.94 & 38 & 8 & 0.66 \\
\cline { 2 - 7 } & 15 & 85.13 & 22.11 & 100 & 19 & 0.47 \\
\hline
\end{tabular}


표 8은 실제 방송 실험을 통해 생성된 손실 데이 터의 버스트 특성을 고려한 $\mathrm{FEC}$ 를 적용하기 위해 수식 (1)을 이용하여 FEC 비율을 결정하고 복원 후 손실율을 나타낸 것이다. 3장에서 랜덤 손실 패턴의 경우 $\mathrm{Gmin}$ 값이 증가 할수록 $\mathrm{FEC}$ 비율이 급격 하 게 증가 하는 것을 확인 할 수 있었다. $12 \mathrm{Mbps}$ 의 경우를 예로 들면 $\mathrm{Gmin}$ 값이 9에서 15로 증가함에 따라 $\mathrm{FEC}$ 블록 크기 $\mathrm{n}$ 값이 2 배로 증가함을 볼 수 있다. 따라서 실제 Wi-Fi 방송 환경에서 발생하는 손실 특성은 랜덤 손실 특성의 성질이 더 높은 것 을 확인 할 수 있다. 또한, 랜덤 손실의 특징을 가 지지만 수식 (1)에서 버스트 손실을 고려한 FEC 복 원 방법을 적용 할 경우에도 높은 복원율을 보이는 것을 확인 할 수 있다.

평균 패킷 손실율을 PLR, 원본 패킷 수를 $\mathrm{k}$, 여 분 패킷 수를 $\mathrm{n}-\mathrm{k}$ 라고 할 때 일반적으로 $\mathrm{FEC}$ Block Erasure Code 기법에서 복원이 이루어지기 위해서는 최소 $\mathrm{k}$ 개 이상이 패킷이 수신 되어야 되 어야 한다. 따라서 $\mathrm{FEC}$ 비율은 $(k /(k+(n-k)))$ 로 나타낼 수 있으므로 수식 (3)과 같다.

$$
\begin{gathered}
\mathrm{k} /(\mathrm{k}+(\mathrm{n}-\mathrm{k}))=1-\mathrm{PLR} \\
(\mathrm{n}-\mathrm{k})=\left(\mathrm{k}^{*} \mathrm{PLR}\right) /(1-\mathrm{PLR})
\end{gathered}
$$

수식 (3)로부터 FEC 여분 패킷의 수 $\mathrm{n}-\mathrm{k}$ 은 수식 (4)에 의해 결정 된다 ${ }^{[13]}$. 수식 (4)에서 확인 할 수 있듯 여분 패킷의 양은 패킷 손실율과 밀접한 관계 가 있다. 따라서 랜덤 손실 특성을 가지는 실제 방 송 패킷 특성에 다음과 같은 수식을 활용하여 $\mathrm{k}$ 가 20 라고 가정 할 때 $\mathrm{FEC}$ 를 적용 하면 다음 표 9와 같다.

표 9 . 버스트 특성을 고려한 FEC 복원율 비교 Table 9. The comparison of FEC recovery rate according to burst loss characteristics

\begin{tabular}{|c|c|c|c|c|}
\hline $\begin{array}{c}\text { Trans- } \\
\text { mission } \\
\text { Rate } \\
\text { (Mbps) }\end{array}$ & $\begin{array}{c}\text { PLR } \\
(\%)\end{array}$ & $\mathrm{n}$ & $\mathrm{n}-\mathrm{k}$ & RPLR \\
\hline 1 & 8.65 & 22 & 2 & 1.80 \\
\hline 6 & 15.48 & 24 & 4 & 2.29 \\
\hline 12 & 16.19 & 24 & 4 & 2.34 \\
\hline 18 & 16.44 & 24 & 4 & 2.57 \\
\hline
\end{tabular}

실제 Wi-Fi 방송 환경에서 발생하는 손실 특성에 수식(4)를 적용하여 $\mathrm{FEC}$ 를 적용 해 본 결과 약 $16 \%$ 의 평균 손실율을 가질 때 복원 후 손실율은 약 $2.5 \%$ 임 것을 확인 할 수 있었다. 즉, 실제 방송 패킷의 손실 특성이 랜덤 손실 특성을 보이기 때문 에 평균 손실율을 고려한 $\mathrm{FEC}$ 를 적용 하여도 패킷 복원이 효율적으로 이루어지는 것을 확인 할 수 있 다. 하지만 실제 방송 패킷에서 버스트 손실의 특성 이 크게 나타나지 않지만 평균 손실을 고려한 FEC 기법 보다 버스트 손실을 고려한 $\mathrm{FEC}$ 적용 기법을 적용 하였을 때 더 높은 복원율을 보이기 때문에 추후 실제 방송 서비스를 제공 하고자 할 때 실제 방송 패킷의 손실 특성을 고려한 효율적인 복원 방 안에 대한 연구가 필요하다.

\section{$\mathrm{V}$. 결 론}

본 논문에서는 $\mathrm{Wi}-\mathrm{Fi}$ 방송 서비스를 제공하기 위 해 실제 무선 랜 환경의 손실 특성 중에서 전송 중 에 데이터가 갑자기 집중적으로 손실이 발생 하는 버스트 손실 특성에 주목하였다. Wi-Fi 전송률에 따 른 버스트 손실 특성을 알아보기 위해 실제 전송률 을 $1 \mathrm{M}-18 \mathrm{Mbps}$ 로 변화 시키면서 실험 하였다. 그리 고 그 결과를 4-상태 마코프 손실 모델을 이용 하 여 버스트 구간과 공백 구간으로 손실을 분리하고 각각의 길이, 밀도, 비율과 같은 특성에 대해서 분 석을 하였다.

또한 실제 $\mathrm{Wi}-\mathrm{Fi}$ 방송 패킷 전송 실험을 통해 생성 된 손실 패턴이 버스트한 특성을 보이는지를 판단하기 위해서 확률 적인 접근으로 분석을 하였 다. 임의의 손실 패킷을 선택했을 때 그 손실 패킷 이 버스트 손실로 평가될 확률을 구하여 전체 손실 패킷 중 버스트 구간에 속한 손실 패킷의 비율과 비교 한 결과 거의 유사한 값을 보였다. 따라서 실 제 무선 랜 환경의 W-Fi 방송 패킷 전송 과정에서 발생하는 손실 패턴은 버스트 손실 특성 보다는 랜 덤 손실에 가까운 특성을 보이는 것을 확인 하였다. 또한 실제 손실 패턴에 버스트 손실을 고려한 FEC 와 평균 손실율을 고려한 $\mathrm{FEC}$ 를 적용 하였을 때 두 방법 모두 높은 복원율을 보임을 확인 하였다.

다양한 관련 연구에서 무선 랜 환경에서 발생하 는 버스트 손실 특성에 대한 연구를 진행 하고 있 다. 실제 본 논문의 실험 환경은 제한 적인 환경과 제한적인 인자로 실험하였기 때문에 추후 $\mathrm{Wi}-\mathrm{Fi}$ 방 송 패킷 전송 과정에서 발생 하는 버스트 손실 특 
성을 보다 정확히 분석하기 위해서는 다양한 환경 을 고려한 실험이 필요하다. 또한, 관련 연구에서 제안하였던 버스트 손실을 고려한 $\mathrm{FEC}$ 인자 결정 방법 또한 $\mathrm{Gmin}$ 값을 고정 하여 유동 적인 손실 복 원을 하기 어렵다. 따라서 추후 버스트 손실을 고려 한 보다 더 효율 적인 $\mathrm{FEC}$ 인자 결정 방법 제시가 요구된다. 또한 추후 연구를 진행 하면서 RS Code 이외에도 터보부호나 LDPC 부호와 같은 타 복원 기법을 고려한 버스트 손실을 복원 연구가 필요하 다.

\section{References}

[1] IEEE 802.11 Working Group, Part 11: Wireless LAN Medium Access Control(MAC) and Physical layer(PHY) specifications, ANSI/IEEE Std. 802.11, Mar. 2007.

[2] L. Rizzo, "Effective erasure codes for reliable computer communication protocols," ACM SIGCOMM Comput. Commun. Review, vol. 27, no. 2, pp. 24-36, Apr. 1997.

[3] T. Friedman, R. Caceres, and A. Clark, "RFC 3611 - RTP Control Protocol Extended Reports (RTCP-XR)," IETF, Nov. 2003.

[4] A. Clark and A. Pendleton, "RTCP XR - IP Video metrics report blocks draft-clark-avt-rtcpxr-video - 00,” IETF (Internet draft expires June 2006), Jan. 2006.

[5] C. Perkins, O. Hodson, and V. Hardman, "A survey of packet loss recovery techniques for streaming audio," IEEE Network, vol. 12, no. 5, pp. 40-48, Sep.-Oct. 1998.

[6] A. Clark, "Modeling the effects of burst packet loss and recency on subjective voice quality," in Proc. IP Telephony Workshop, pp. 123-127, Geneva, Switzerland, Mar. 2001.

[7] Y. J. Liang, J. G. Apostolopoulos, and B. Girod, "Analysis of packet loss for compressed video: does burst-length matter?," in Proc. IEEE Int. Conf. Acoust. Speech Signal Process. (ICASSP), vol. 5, pp. V-684-7, Hong Kong, China, Apr. 2003.

[8] C.-H. Lin, C.-H. Ke, C.-K. Shieh, and N. K. Chilamkurti, "The packet loss effect on MPEG video transmission in wireless networks," in
Proc. $20^{\text {th }}$ Int. Conf. Advanced Inform. Networking Applicat. (AINA), pp. 565-572, Vienna, Austria, Apr. 2006.

[9] M. Johanson "Adaptive forward error correction for real-time internet video," in Proc. 13th Int. Packet Video Workshop, pp.196 -205, Nantes, France, Apr. 2003.

[10] A. Nafaa, T. Ahmed, and A. Mehaoua "Unequal and interleaved FEC protocol for robust MPEG-4 multicasting over wireless LANs," in Proc. IEEE Conf. Commun. (ICC), vol. 3, pp. 1431-1435, Paris, France, June 2004.

[11] G. Convertino and S. L. Oliva, "Adaptive FEC for 802.11 burst losses reduction," in Proc. $2^{\text {nd }}$ Int. Mobile Multimedia Commun. Conf. (MobiMedia), Article no. 20, Alghero, Italy, Sep. 2006.

[12] G. Convertino, S. L. Oliva, F. Sigona, and L. Anchora, "MMC05-1: an adaptive FEC scheme to reduce bursty losses in a 802.11 network," in Proc. IEEE Global Telecommun. Conf. (GLOBECOM), pp. 1-6, San Francisco, U.S.A., Nov.-Dec. 2006.

[13] O. Alay, T. Korakis, Y. Wang, and S. Panwar, "An experimental study of packet loss and forward error correction in video multicast over IEEE $802.11 \mathrm{~b}$ network," in Proc. $6^{\text {th }}$ IEEE Consumer Commun. Networking Conf. (CCNC), pp. 1-5, Las Vegas, U.S.A., Jan. 2009.

[14] S.M. Kim, D.H. Kim, and J.D. Kim, "A study of the quality measurement considering Burst Loss in Wi-Fi broadcasting system," in Proc. KICS Int. Conf. Commun. (KICS ICC), pp. 463-464, Jeju Island, Korea, June 2012.

\section{김 세 미 (Se-mi Kim)}

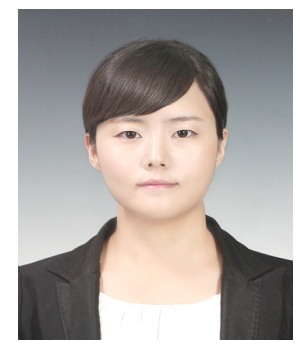

2011년 2월 부산대학교 정보 컴퓨터공학부 졸업

2013년 2월 부산대학교 컴퓨 터공학과 석사

<관심분야> 무선통신 
김 동 현 (Dong-hyun $\mathrm{Kim}$ )

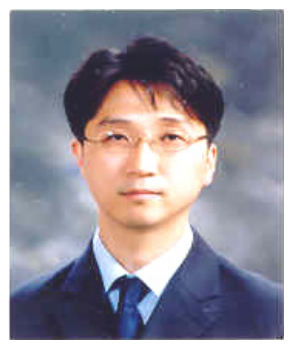

1998년 2월 동의대학교 공과대

학 전자통신공학과 졸업

2004년 2월 동의대학교 대학

원 정보통신공학과 석사

2008년 3월 현재 부산대학교

컴퓨터공학과 박사과정

<관심분야> 무선통신

\section{김 종 덕 (Jong-deok Kim)}

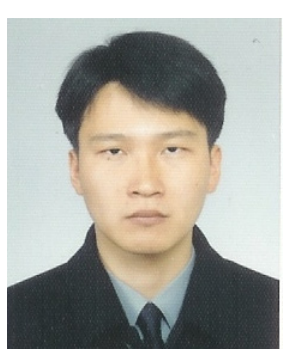

1994년 2월 서울대학교 계산통 계학과 졸업

1996년 2월 서울대학교 전산과

학과 석사

2003년 2월 서울대학교 컴퓨터

공학과 박사

2004년 2월 현재 부산대학교

정보컴퓨터공학부 부교수

<관심분야> 무선통신 